\title{
GENERALIZED MULTISCALE CONNECTED OPERATORS WITH APPLICATIONS TO GRANULOMETRIC IMAGE ANALYSIS
}

\author{
Anastasios Doulamis, Nikolaos Doulamis, and Petros Maragos \\ National Technical University of Athens, \\ Dept. of Electrical and Computer Engineering, Athens 15773, Greece \\ Email: [adoulam, ndoulam, maragos] @es.ntua.gr
}

\begin{abstract}
In this paper, generalized granulometric size distributions and size histograms (a.k.a 'pattern spectra') are developed using generalized multiscale lattice operators of the opening and closing type. The generalized size histograms are applied to granulometric analysis of soilsection images. An interesting structure is obtained when the histogram is based on area openings. Furthermore, a fast implementation of the generalized size histograms is presented using threshold analysis-synthesis. Comparisons with size distributions based on conventional morphological operators indicate that the generalized histograms provide a more direct and informative description of the image content in objects with scale-dependent geometric attributes. Applications are also developed for studying the structure of soilsection images.
\end{abstract}

\section{INTRODUCTION}

Nonlinear multiscale image analysis has recently emerged as a useful tool for many applications in image processing and computer vision, where it performs better than linear (Gaussian) scale-space. Examples include geometry-based filtering with better edge preservation, motion tracking, efficient image segmentation, shape analysis, measurements and modeling of fractal signals and granulometric image analysis (size distributions).

The classic morphological multiscale analysis with openings and closings [5, 7] provides us with useful descriptors such as the size distributions and their corresponding size densities (histograms), called 'pattern spectrum' in [3], which can detect critical scales of image objects and quantify the multiscale shape-size content of an image. These tools have been applied successfully to a variety of image analysis tasks, such as feature extraction, texture characterization, shape-size analysis, object recognition both for binary and grayscale images $[5,7,3,1]$. Application domains include the fields of geological, biomedical, and document image analysis. Traditionally, the size distributions are formed by computing the areas or volumes of standard morphological openings and closings (i.e. compositions of Minkowski erosions and dilations) by convex structuring elements (e.g. disks or lines) at multiple scales. However, this conventional approach has weak points because the standard openings do not retain the contours of image objects, cannot directly localize important image information such as the area

This research work was supported by the Greek Secretariat for Research and Technology and by the European Union under the program חENE $\Delta-$ 99 with Grant \# 99E $\Delta 164$. of its connected components, and are not oriented toward objectbased analysis. Furthermore, the information they provide about the image shape-size content is spread among different scales and hence is not directly useful for object-oriented analysis.

In this paper, the aforementioned difficulties are addressed by using size distributions and corresponding size histograms based on generalized multiscale openings. These can be formalized using the theory of image operators on complete lattices [2]. One class of generalized openings we use are the reconstruction openings $[8,6]$ which can reconstruct whole objects (marked by some seed) with extract preservation of their contour; in this reconstruction process they simplify the original image by completely eliminating all objects inside which the marker cannot fit. Another interesting class of generalized operators are the area openings [9] which filter connected components of an image according to their area. Both the reconstruction and the area openings are connected operators; hence they are suitable for object-oriented size analysis.

In this paper we present a generalized granulometric image analysis based on multiscale reconstruction and area openings and closings. We study some theoretical properties of these generalized size histograms and apply them to studying the multiscale structure of soilsections based on their images. Analysis of this kind of images is important since it helps ecologists and biologists to measure various aspects of ground quality and understand the type and degree of changes that a terrestrial ecosystem has undergone.

\section{GENERALIZED MULTISCALE OPERATORS}

Consider the complete lattice $\mathcal{L}$ of real-valued image signals equipped with the partial ordering $f \leq g[f(x) \leq g(x) \forall x]$, the supremum $\bigvee$ and the infimum $\Lambda$. Let $\alpha_{s}$ and $\beta_{s}$ denote families of multiscale operators on $\mathcal{L}$ of the opening and closing type, respectively, which depend on a scale parameter $s \geq 0$. For $\alpha_{s}$ to be a lattice opening, it should satisfy three properties: increasing: $f \leq g \Longrightarrow \alpha_{s}(f) \leq \alpha_{s}(g)$, antiextensive: $\alpha_{s}(f) \leq f$, and idempotent: $\alpha_{s}\left(\alpha_{s}(f)\right)=\alpha_{s}(f)$, for any image signal $f$ and any scale $s$. Similarly, $\beta_{s}$ is a lattice closing if it is increasing, idempotent and extensive. The last property means that $\beta_{s}(f) \geq f$.

Furthermore, as the scale $s$ varies, we require from these families of multiscale openings and closings to be monotonic:

$$
s<r \Rightarrow\left\{\begin{array}{l}
\alpha_{s}(f) \geq \alpha_{r}(f) \\
\beta_{s}(f) \leq \beta_{r}(f)
\end{array}\right.
$$

Note that, at scale $s=0$, both the opening and the closing become the identity operator; i.e., $\alpha_{0}(f)=\beta_{0}(f)=f$. Property (1) 
indicates that, as the scale increases, the output of the generalized multiscale opening (closing) does not increase (decrease). Based on the above, we can unify large classes of operators that share a few fundamental properties as generalized multiscale open-closings. In the following, some interesting special cases are presented.

\subsection{Conventional Multiscale Open-Closing Operators}

The conventional multiscale opening and closing operators are generated by compositions of Minkowski erosions and dilations of the signal $f$ by a flat scale-parametric structuring element $s B$ :

$$
\begin{aligned}
& \alpha_{s}(f)=(f \ominus s B) \oplus s B \\
& \beta_{s}(f)=(f \oplus s B) \ominus s B
\end{aligned}
$$

where $\oplus$ and $\ominus$ denote the Minkowski morphological dilation and erosion, respectively. In the continuous case (images defined on $\mathbb{R}^{2}$ ), the above multiscale open-closings satisfy the monotonicity property (1) if $B$ is a compact convex set and $s B=\{s b: b \in B\}$; typically, $s B$ is a disk of radius $s$. In this case, the scale is defined by the size (radius) $s$ of the structuring element. In the discrete case (images defined on $\mathbb{Z}^{2}$ ), the multiscale open-closings satisfy (1) if $s B$ is obtained by dilating the unit-size $B$ with itself $s$ times.

\subsection{Multiscale Open-Closing by Reconstruction}

The reconstruction opening (closing) reconstructs the parts from the foreground (background) of an image $f$ that are hit by a marker signal $m$. For the reconstruction opening (closing) we must have $m \leq$ $f(m \geq f)$. To produce multiscale openings/closings by reconstruction we make the marker signals to be scale-dependent. The simplest way (adopted in this paper) is to use as marker for the reconstruction opening the multiscale Minkowski erosion $m_{s}^{-}(f)=$ $f \ominus s B$ of the original image $f$. Similarly, as marker for the multiscale reconstruction closing we use the multiscale dilation of $f$ by a disk: $m_{s}^{+}(f)=f \oplus s B$.

Consider the conditional dilation and erosion of the markers $m_{s}(f)$ by the unit disk $B$ with reference the signal $f$ :

$$
\begin{aligned}
& \delta_{B}\left(m_{s}^{-}(f) \mid f\right)=\left(m_{s}^{-}(f) \oplus B\right) \wedge f \\
& \varepsilon_{B}\left(m_{s}^{+}(f) \mid f\right)=\left(m_{s}^{+}(f) \ominus B\right) \vee f
\end{aligned}
$$

Then, one algorithm to obtain the multiscale reconstruction opening (closing) of $f$ from the corresponding marker $m_{s}$ is by iterating the above conditional dilation (erosion):

$$
\begin{aligned}
& \alpha_{s}(f)=\lim _{n \rightarrow \infty} \delta_{B}^{n}\left(m_{s}^{-}(f) \mid f\right)=\bigvee_{n \geq 1} \delta_{B}^{n}\left(m_{s}^{-}(f) \mid f\right) \\
& \beta_{s}(f)=\lim _{n \rightarrow \infty} \varepsilon_{B}^{n}\left(m_{s}^{+}(f) \mid f\right)=\bigwedge_{n \geq 1} \varepsilon_{B}^{n}\left(m_{s}^{+}(f) \mid f\right)
\end{aligned}
$$

where $\psi^{n}$ denotes the $n$-fold composition of an operator $\psi$ with itself. Since the reconstruction opening and closing are increasing with respect to the marker, they satisfy property (1).

\subsection{Multiscale Area Open-Closing}

The area open-closing is another class of generalized morphological operators. These filters suppress arbitrarily-shaped connected components in an image whose areas (number of pixels) are smaller than a given threshold (scale) $s$. Therefore, in this case, the scale is directly related to an object property, which provides a more physical interpretation of the image content. The area opening can easily be implemented in binary images: let the set $X=\bigsqcup_{i} C_{i}$ represent a binary image, where $C_{i}$ represent the connected components of $X$. The area opening output is $\alpha_{s}(X)=\bigsqcup_{j} C_{j}$ with area $\left(C_{j}\right) \geq s$, $\forall j$. Any increasing binary operator can be extended to graylevel images via threshold superposition [4]. Consider a graylevel image $f$ and its threshold binary signals $f_{h}(x)$ where $h$ ranges over all gray levels. The value of $f_{h}(x)$ is 1 if $f(x) \geq h$ and 0 otherwise. Then, the graylevel area opening is defined via threshold superposition [9] as $\alpha_{s}(f)(x)=\sup \left\{h: \alpha_{s}\left(f_{h}\right)(x)=1\right\}$. If the image $f$ takes only nonnegative integer values $\{0,1, \ldots, m\}$, then

$$
\alpha_{s}(f)=\sum_{h=1}^{m} \alpha_{s}\left(f_{h}\right)
$$

Similarly, we can define the area closing of $f$ by duality as $\beta_{s}(f)=$ $m-\alpha_{s}(m-f)$. Since the area of a set is an increasing operator, the area opening and closing satisfy property (1).

The threshold decomposition scheme enabled us to develop a more efficient and fast implementation of the multiscale area openclosing compared to the (single-scale) method of [9]. This is due to the fact that the image threshold decomposition and the area of the connected components are estimated only once. Then, at each scale, a simple comparison of the area of the connected components to the scale $s$ is performed. Instead, the method of [9] needs reestimation of the area operator at every scale.

\subsection{Generalized Size Histogram (Pattern Spectrum)}

By applying a generalized opening or closing operator to an image $f$ for all different scales $s$ we can create the granulometry (which is closely related to a size distribution)

$$
G_{f}(s)= \begin{cases}\operatorname{Vol}\left(\alpha_{s}(f)\right), & s \geq 0 \\ \operatorname{Vol}\left(\beta_{-s}(f)\right), & s<0\end{cases}
$$

where $\operatorname{Vol}(f)=\int f(x) d x$ is volume under the surface of $f$. Due to (1), the granulometry $G_{f}(s)$ decreases as $s$ increases: $s<r \Longrightarrow$ $G_{f}(r) \leq G_{f}(s)$. Further, it can be shown that [3], for images $f$ with a compact support, there is a scale $s_{\min }<0$ such that $\beta_{-s}(f)=\beta_{-s_{\text {min }}}(f) \forall s<s_{\text {min }}$. Similarly, there is a scale $s_{\max }>0$ such that $\alpha_{s}(f)=0 \forall s>s_{\max }$. Therefore, the normalized negated granulometry $D_{f}(s)=1-G_{f}(s) / G_{f}\left(s_{\text {min }}\right)$ behaves like the size distribution of a random variable whose value is related to the size content of $f$. The derivative of this distribution yields a size density which behaves like the probality density function of this random variable. Ignoring in this size density, for notational simplicity, the normalizing factor $G_{f}\left(s_{\min }\right)$ yields a nonnegative function

$$
P_{f}(s)=-d G_{f}(s) / d s
$$

This unnormalized size density is also called 'pattern spectrum' due to its ability to quantify the shape-size content of images [3]. Since the granulometry decreases monotonically, $P_{f}(s) \geq 0, \forall s$.

For discrete images $f$, we use integer scales $s=0$, the granulometry $G_{f}(s)$ is obtained as above by defining $\operatorname{Vol}(f)$ as the sum of values of $f$, and the size density $P_{f}(s)$ is obtained by using differences instead of derivatives: $P_{f}(s)=G_{f}(s)-G_{f}(s+1)$. In the discrete case, we call $P_{f}(s)$ a size histogram.

Both the standard openings used in this paper as well as the reconstruction and area openings (and the corresponding closings) are flat operators that obey the threshold superposition. As shown in 
[3] for the case of standard openings, the pattern spectrum inherits this property. The proof of this property easily extends to the case of the reconstruction and area openings. Thus, if a discrete image $f$ assumes only nonnegative integer values $\{0,1, \ldots, m\}$, then

$$
P_{f}(s)=\sum_{i=1}^{m} P_{f_{i}}(s)
$$

where $f_{i}$ is the threshold binary image obtained from $f$ by thresholding it at level $i$. The above property allowed us to develop a fast algorithm for measuring the generalized size histograms, because the size histograms based on reconstruction and area openings become extremely fast when applied to binary images since we essentially need just to label the connected components of the binary image and count their areas. Then the total size histogram results as the sum of the histograms of all the threshold binary images.

\subsection{A Property of Area Size Histogram}

Assume first that $f$ is a binary image. Then, it is straightforward to show that its size histogram based on area openings is

$$
P_{f}(s)=s N(s), \quad N(s)=\# \text { of conn. components with area } s
$$

For graylevel images we have shown that their area size histogram has the form

$$
P_{f}(s)=s \sum_{i=1}^{N(s)} h_{i}(s)
$$

where $h_{i}(s)$ is the height difference between a regional maximum (or a crossection of $f$ whose area became larger than the previous higher crossection) and its highest neighbor around the boundary of this plateau, and $N(s)$ is the number of such plateaus with area $s$. For an image $f$ with integer values, the above property of the area size histogram implies that, the area-normalized histogram $P_{f}(s) / s$ has integer values which count the number of connected components (for binary images) or of regional maxima (for graylevel images) with area $s$. This theoretical property allows us to detect the presence of connected components of any specific area, or zone of area scales, in an image. Instead, the contribution of one connected component to the conventional pattern spectrum is usually spread out, which does not facilitate its accurate detection. This property of the area histogram is verified in Figs. 3, 4.

\section{APPLICATION TO SOILSECTION IMAGES}

The aforementioned granulometric analysis based on generalized openings is applied to the characterization and description of the size content of soilsection images. In analyzing such images it is of great interest to find soil characteristics or features that can be detected or measured automatically and then coresponded with the biochemical properties of the soil. Such a procedure can lead to an automated system for evaluating the bioecological quality of soil by analyzing images of soilsections.

Figure 1(a) illustrates a soilsection image; the white regions correspond to air voids, while the dark regions to soil pedofeatures or aggregates. Its size histogram based on conventional morphological filters is shown in Fig. 1(b). As expected, this openingclosing histogram has a complex graph whose large spikes might indicate the existence of objects with components at those scales. In Fig. 1(c) we show another histogran based on multiscale erosions/dilations by disks. The erosion-dilation histogram is not as informative and simply decreases with scale; the slope of this decrease might be of some importance since it is related to the Miknowski fractal dimension of the image surface.

Figure 2 illustrates the size distribution and histogram based on openings/closings by reconstruction for the soilsection image of Fig. 1(a). As can be seen, a much better localization of the object size is obtained, in the sense that the histogram presents abrupt peaks, especially at the scales where large connected objects are localized. Figure 3 shows the results of the granulometric analysis for the image of Fig. 1(a) based on area closings. As observed, the size histogram presents a highly localized behavior (there are lots of isolated spikes, especially at large areas), while its values increase proportionally to the area. A zoom in Fig. 3(c) reveals that, all peaks of the area-normalized histogram have integer values and most of them are equal to one. Likewise in Fig. 4 for another soilsection image. This experimentally confirms the theoretical property mentioned in Sec. 2.5 .

In conclusion, the generalized size histograms based on reconstruction and area openings/closings yield a clearer (locally more concentrated) spike distribution and hence are more informative than histograms based on conventional openings. Thus, spikes in the generalized size histograms directly correspond with the existence of connected image components with a specific geometric property (i.e., radius of maximum inscribable disk or area), whereas the contribution of one component to the conventional histogram is usually spread out. Area size histograms can even count the number of distinct components with a specific area. In general, size histograms are useful for analyzing size content in images with a large number of components in the foreground and/or background. Soilsection images are a such a typical class where granulometric analysis yields very useful information. Specifically, it can provide estimates of: 1) the average size of grains and pores, 2) deviation around the mean or other moments, 3) the percent of grains/pores in localized scale zones, 4) the coarse-to-fine ratio, 5) the geometrical complexity of grain/pore size distribution, 6) all the above with various alternative interpretations of 'scale' based on different geometrical properties (e.g., smallest or largest diameter, area, degree of connectivity).

\section{REFERENCES}

[1] E.R. Dougherty, J. Newell and J. Pelz, "Morphological Texture-based Maximum-Likelihood Pixel Classification based on Local Granulometric Moments", Pattern Recognition, vol.25, Nov. 1992.

[2] H. Heigmans, Morphological Image Operators, Acad. Press, New York, 1994.

[3] P. Maragos, "Pattern Spectrum and Multiscale Shape Representation" IEEE Trans. Pattern Anal. Machine Intellig., vol. 11, pp. 701-716, 1989.

[4] P. Maragos and R. D. Ziff, "Threshold Superposition in Morphological Image Analysis Systems", IEEE Trans. Pattern Analysis and Machine Intell., vol.12, pp.498-504, May 1990.

[5] G. Matheron, Random Sets and Integral Geometry, Wiley, New York, 1975.

[6] P. Salembier and J. Serra, "Flat Zones Filtering, Connected Operators, and Filters by Reconstruction", IEEE Trans. Image Processing, vol. 4, pp.11531160, Aug. 1995

[7] J. Serra, Image Analysis and Mathematical Morphology, Acad. Press, New York, 1982.

[8] L. Vincent, "Morphological grayscale reconstruction in image analysis: Applications and efficient algorithms", IEEE Trans. Image Procesing, vol. 2, no. 2 , pp. 176-201, 1993.

[9] L. Vincent, "Morphological area openings and closings for grayscale images," Proc NATO Workshop on "Shape in Picture,", Driebengen, The Netherlands, Spinger-Verlag, pp. 197-208, Sep. 1992. 


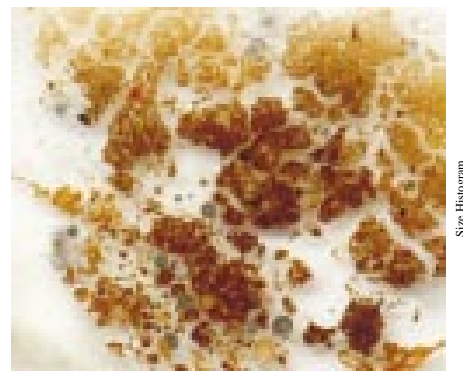

(a)

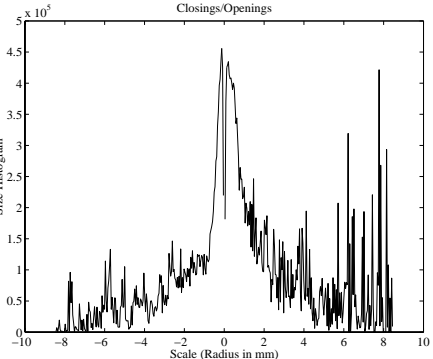

(b)

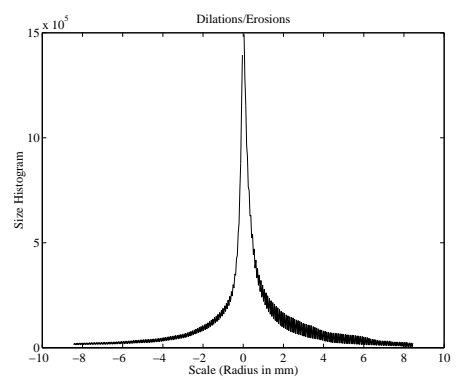

(c)

Fig. 1. Conventional size histograms for a soil section image. (a) Original image (405x479 pixels, 20.3x17.2mm). (b) Size histogram based on flat Minkowski openings/closings. (c) Histogram based on multiscale erosions/dilations.

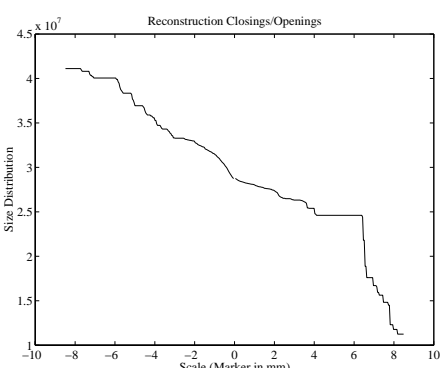

(a)

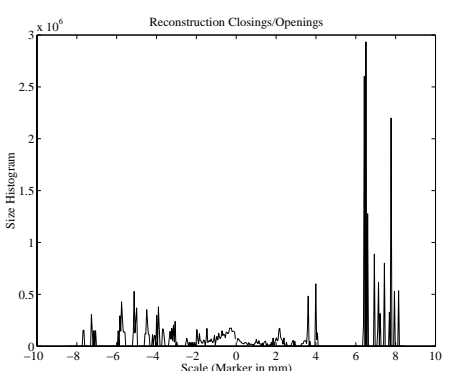

(b)

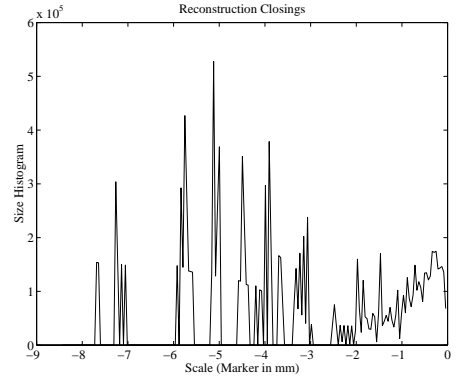

(c)

Fig. 2. Granulometric analysis for the image of Figure 1(a) based on opening-closing by reconstruction. (a) Size distribution. (b) Size histogram. (c) A zoom of the size histogram.

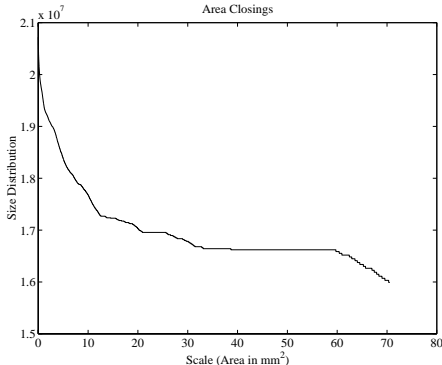

(a)

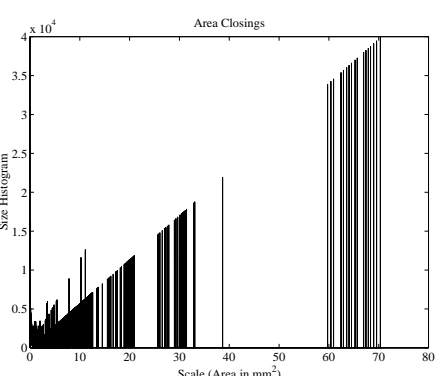

(b)

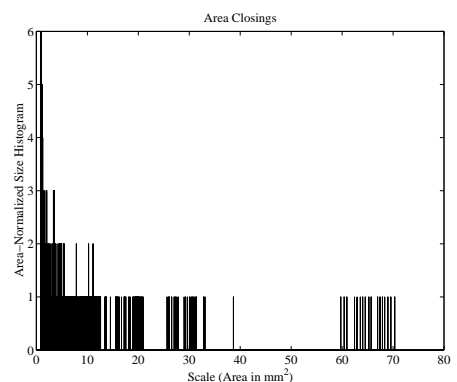

(c)

Fig. 3. Granulometric analysis using area closings on the image of Fig. 1(a). (a) Size distribution. (b) Size histogram. (c) Size histogram normalized per area.

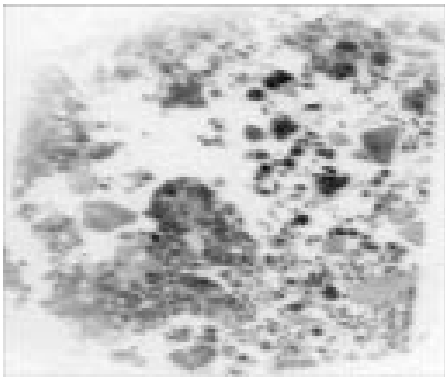

(a)

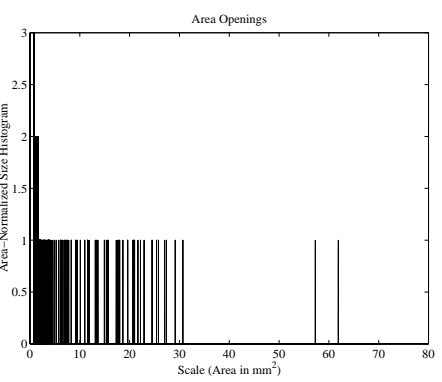

(b)

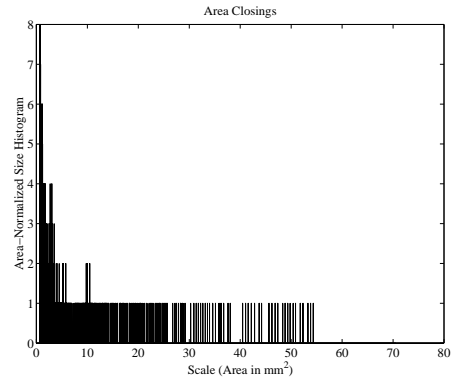

(c)

Fig. 4. (a) Original image $(584 \times 447$ pixels, $18.9 \times 24.7 \mathrm{~mm})$. (b) Normalized size histogram based on area openings. (c) Normalized size histogram based on area closings. 